\section{BNT211: A PHASE I/II TRIAL TO EVALUATE SAFETY AND EFFICACY OF CLDN6 CAR-T CELLS AND VACCINE- MEDIATED IN VIVO EXPANSION IN PATIENTS WITH CLDN6-POSITIVE ADVANCED SOLID TUMORS}

${ }^{1}$ Andreas Mackensen*, ${ }^{2}$ Christian Koenecke, ${ }^{3} J o h n$ Haanen, ${ }^{4}$ Winfried Alsdorf, ${ }^{5}$ Alexander Desuki, ${ }^{5}$ Eva Wagner-Drouet, ${ }^{6}$ Daniel Heudobler, ${ }^{7}$ Peter Borchmann, ${ }^{8}$ Erol Wiegert, ${ }^{9}$ Catrine Schulz, ${ }^{9}$ Benjamin Rengstl, ${ }^{9}$ Liane Preussner, ${ }^{9}$ Oezlem Tuereci, ${ }^{9}$ Ugur Sahin. 'University Hospital Erlangen, Erlangen, NE, Germany; ${ }^{2}$ Hannover Medical School, Hanover, Germany; ${ }^{3}$ Netherlands Cancer Institute, Amsterdam, Netherlands; ${ }^{4}$ University Medical Center Eppendorf, Hamburg, Germany; ${ }^{5}$ University Medical Center Mainz, Mainz, Germany; ${ }^{6}$ University Hospital Regensburg, Regensburg, Germany; ${ }^{7}$ University Hospital of Cologne, Cologne, Germany; ${ }^{8}$ Bexon Clinical Consulting, Upper Montclair, USA; ${ }^{9}$ BioNTech SE, Mainz, Germany

Background BNT211 is a chimeric antigen receptor (CAR)-T cell product candidate that targets the tumor specific antigen Claudin-6 (CLDN6). Preclinical studies demonstrated that combining these engineered cells with a CAR-T cell Amplifying RNA Vaccine (CARVac) leads to in vivo expansion of adoptively transferred CAR-T cells, resulting in their improved persistence and functionality.

Methods This first-in-human, open label, multi-center trial involves a bifurcated $3+3$ design with separate CLDN6 CAR$\mathrm{T}$ cell dose escalations (single flat-dose) for monotherapy (part 1) and the combination with CARVac (part 2) based on 3 dose levels (DL). In part 2, CARVac is applied every 3 weeks starting at day 4 post transplantation including a one-step intra-patient dose escalation. Patients with CLDN6-positive relapsed or refractory solid tumors without further standard treatment options and ECOG 0 or 1 are eligible for recruitment.

Results As of July 23rd 2021, 8 patients have been treated. DL1 of part 1 has been completed, while dosing of part 1 DL2 and part 2 DL1 is ongoing. One patient with cancer of unknown primary was treated with a dose below DL1 in combination with CARVac; the underlying diseases of the other 7 treated patients were testicular, ovarian and endometrial cancer as well as soft-tissue sarcoma. No acute or doselimiting toxicities and no serious adverse events related to the drug product have been reported. Manageable cytokine release syndrome (CRS, grade 1-2, the latter managed with Tocilizumab) without any signs of neurotoxicity have been observed in both patients of part 1 DL2. Only transient and moderate elevations of IL-6 and CRP serum levels occurred in remaining patients. Notably, administration of CARVac resulted in transient flu-like symptoms resolving within 24h. Analysis of CAR-T cell frequency in peripheral blood revealed robust engraftment followed by decline after day 17 . Further expansion was noted in two patients with liver metastases accompanied by elevated levels of ALT, AST and AP, while total bilirubin was not affected. First tumor assessment 6 weeks after transplantation available for $5 / 8$ patients revealed 4 SD (3 transitioned into PD after an additional 6-18 weeks) and 1 PD. Strikingly, three patients showed initial tumor shrinkage according to RECIST1.1 (reduction of target sum: $-18 \%$, $21 \%$ and $-27 \%)$.

Conclusions CLDN6 CAR-T cells +/- CARVac show a favorable safety profile at doses tested and encouraging signs of efficacy. Updated data from open cohorts and especially for combination with CARVac will be presented.

Acknowledgements BNT211-01 is funded by BioNTech Cell \& Gene Therapies GmbH.

Trial Registration ClinicalTrials.gov: NCT04503278

\section{REFERENCES}

N/A

Ethics Approval Ethics \& Institutional Review Board approvals were obtained from the respective participating countries prior to initiation of the trial.

\section{Consent N/A}

http://dx.doi.org/10.1136/jitc-2021-SITC2021.958 